\title{
Study of Odor Preconcentrator Using SAW Device
}

\author{
Yasufumi Yokoshiki and Takamichi Nakamoto \\ Department of Physical Electronics, Graduate School of Science and Engineering, Tokyo Institute of \\ Technology, 2-12-1 Ookayama, Meguro-ku, Tokyo 152-8550, Japan, \\ yokoshiki@mn.ee.titech.ac.jp
}

\begin{abstract}
:
A new type preconcentrator was realized by using a SAW device and peltier devices. First, the odor is delivered to preconcentrator, in which the peltier device cools the surface of SAW device to accumulate the odor. Then the odor is atomized by acoustic streaming using the SAW device. As a result, we obtained much higher response to 1-nonanol, one of low-volatile compounds, because of preconcentrator effect.
\end{abstract}

Key words: Preconcentrator, SAW device, acoustic streaming, odor sensing system, QCM sensor and peltier device

\section{Introduction}

We have studied an odor sensing system composed of multiple QCM (Quartz Crystal Microbalance) sensors with different coatings and a pattern recognition technique [1-2]. In addition to them, a preconcentrator is often used to raise the sensitivity of the odor sensing system [3-4]. In a conventional preconcentrator, odorant molecules are accumulated at adsorbents in a preconcentrator tube. Then, the accumulated odor is thermally desorbed to raise its concentration so that the sensing system can easily detect the odor with low concentration.

We proposed a new method without adsorbents. Using the conventional preconcentrator tube, it takes time to desorb odorant molecules. Moreover, it is difficult to make several preconcentrator tubes with the same characteristics. The proposed preconcentrator desorbs gas molecules using acoustic streaming effect [5] caused by a SAW (Surface

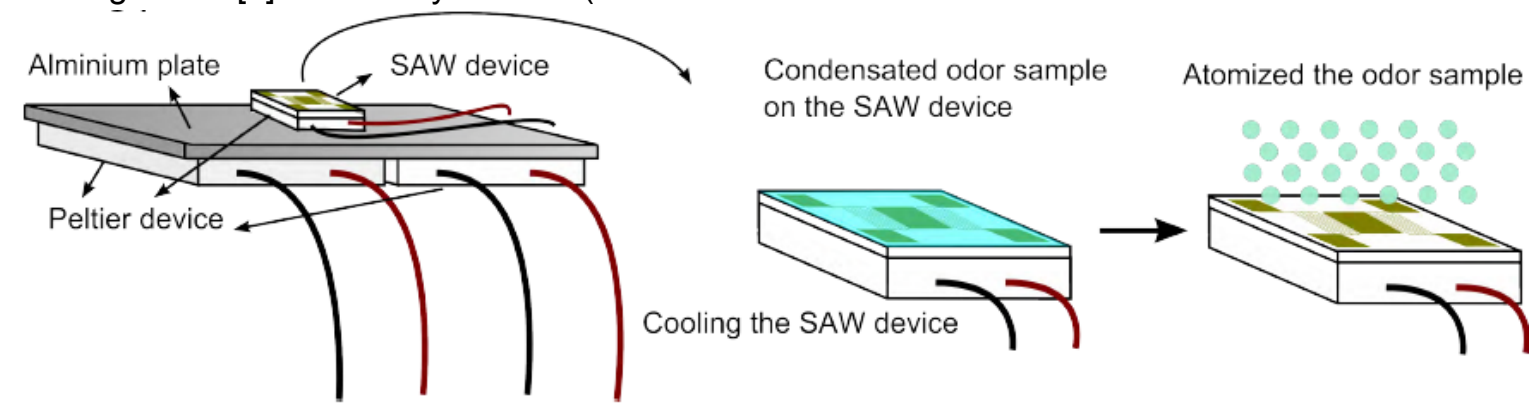

Acoustic Wave) device. The desorption is very rapid since thin liquid layer on a planar device is instantaneously atomized. Moreover, characteristic variation from device to device is negligibly small because they are fabricated using photolithography. The purpose of this study is to investigate the soundness of the basic idea about a new-type preconcentrator.

\section{Principle}

Figure. 1 shows a basic concept of a proposed preconcentrator using SAW device and peltier devices. Although a pump and liquid-droplet handling have been already reported [6], the application of SAW streaming to a preconcentrator device is novel. The peltier devices cool target odor sample and condensate it on a surface of the SAW device, whereas a typical preconcentrator has a heater and adsorbent. Then high-concentration gas is obtained by atomizing it. Atomization occurs owing to acoustic streaming phenomenon.

Fig. 1: Basic concept of proposed preconcentrator. 
Then, we show the cooling temperature required for the preconcentrator device. The relationship between the temperature $[\mathrm{K}]$ and saturated vapor pressure $P_{\text {sat }}[\mathrm{Pa}]$ is expressed as

$\ln \left(P_{\text {sat }}\right)=\frac{c}{T}+d$,

where $c$ and $d$ are constants. $c$ and $d$ can be determined using the data from the handbook [7]. When we calculated linear fatty alcohol as examples, the relationship between $T$ and $P_{\text {sat }}$ is depicted in Fig.2.

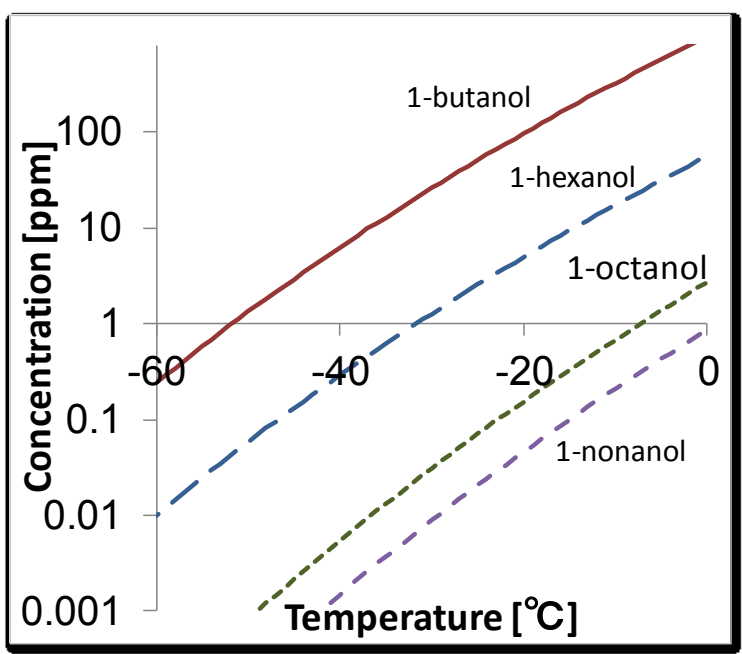

Fig.2 Relationship between temperature and saturated concentration.

The target concentration to be detected is human olfactory threshold [8]. They are tabulated in Table 1. It was found from Table 1 that this preconcentrator is helpful to detect a compound with low volatility. The compound with low volatility is important since a human is very sensitive to it in spite of its low concentration.

Table 1 Temperature required for preconcentration

\begin{tabular}{c|c|c}
\hline Vapor & $\begin{array}{c}\text { Olfactory } \\
\text { threshold } \\
{[\mathrm{ppb}]}\end{array}$ & $\begin{array}{c}\text { Temperature } \\
\text { [Celcius] }\end{array}$ \\
\hline 1-butanol & 490 & -56 \\
\hline 1-hexanol & 44 & -52 \\
\hline 1-octanol & 5.7 & -40 \\
\hline 1-nonanol & 71 & -18 \\
\hline
\end{tabular}

\section{Experimental setup}

The size of SAW device is $1.1 \mathrm{~cm} \times 1.1 \mathrm{~cm}$. Two IDTs (InterDigtal Transducers) are placed on both sides of the atomization area. Each IDT has 10 pairs of fingers and its resonant frequency was $60.9 \mathrm{MHz}$. Two 100 pairs of reflectors are placed outside IDTs. SAW propagates along $X$ direction on $128^{\circ}$ rotated $Y$ CUT LiNbO ${ }_{3}$ substrate.

Fig. 3 shows the photo of the sensor cell. The housing of a sensor cell is made of acrylic resin and a printed circuit board. The cell was fixed using epoxy adhesive. The peltier devices (STS, T151-40-017(s)) is located backside of the printed circuit board and the heat generated from that device with a heat sink was excluded using a fan

In the cell, QCM sensor (Sensing film: Siponate DS-10, 20MHz, AT-CUT) is placed just above SAW device.

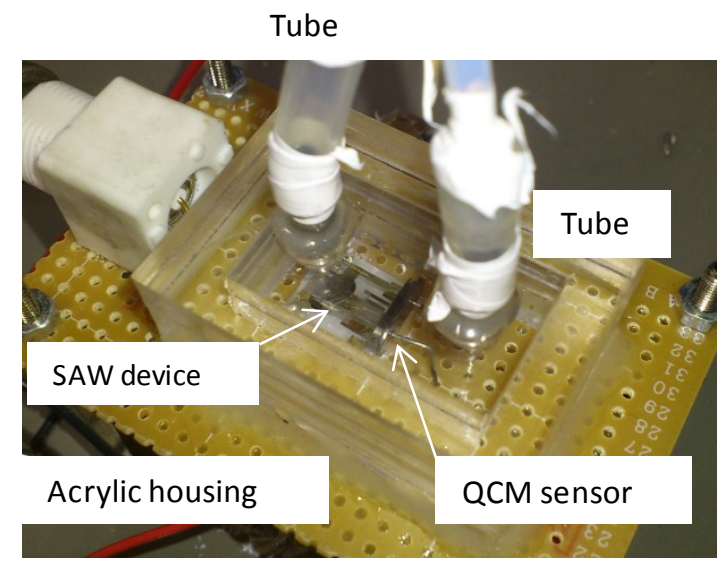

Fig.3 Photo of preconcentrator and sensor cell.

Block Diagram of a measurement is shown in Fig. 4. The green block is implemented into a field programmable gate array (FPGA). The odor was delivered to the SAW device from either sampling bag with dry air or that with odor sample, which was switched by a solenoid valve controlled by FPGA. The flow rate was kept at $200 \mathrm{ml} / \mathrm{min}$. The SAW device atomized the odor accumulated at this surface of SAW device cooled by the peltier devices. Finally, QCM sensor detected the odor in the form of frequency shift after the peltier devices were switched off. The SAW device was driven by RF burst voltage (100Vp-p) from FPGA via RF amplifier (ZHL-5W-1H, Mini-Circuits ). The SAW device, the peltier device, the fan, the solenoid valve, the duty ratio $(1 \%)$ and repetition frequency $(10 \mathrm{~Hz})$ of $R F$ burst wave were controlled by PC via FPGA. 


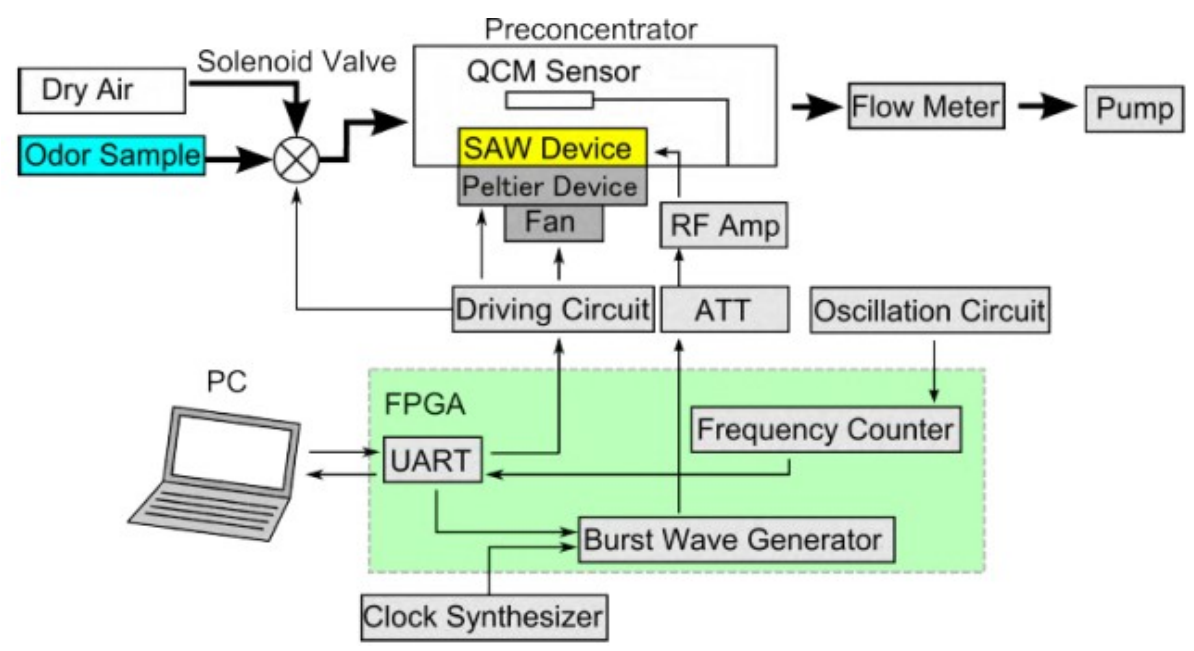

Fig.4 Block diagram of measurement system.

\section{Experimental result}

We studied the preconcentrator separated from the sensor cell as is shown in Fig.5 (a). Fig.5 (b) shows the cell structure the same as that in Fig.4. The preconcentrator and the sensor are located in the same housing. Two types of the cells were compared in this study.

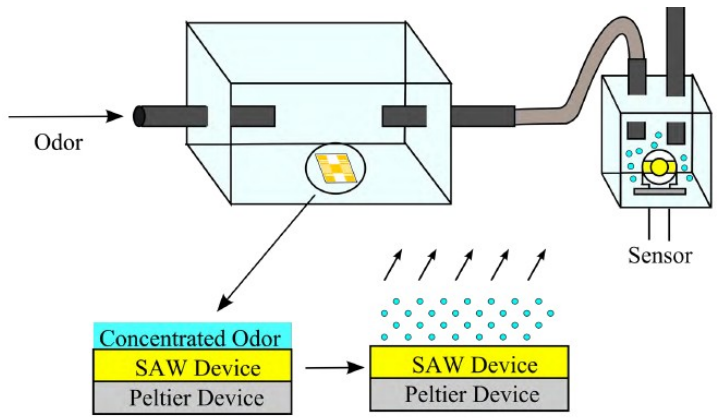

(a) Preconcentrator separated from sensor cell.

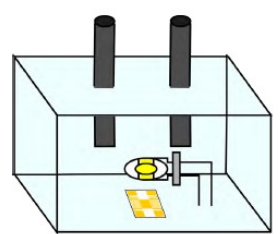

(b) Preconcentrator together with sensor in the same housing.

Fig.5 Cell structure (a) Preconcentrator separeated from sensor cell and (b) preconcentrator together with sensor in the same housing.

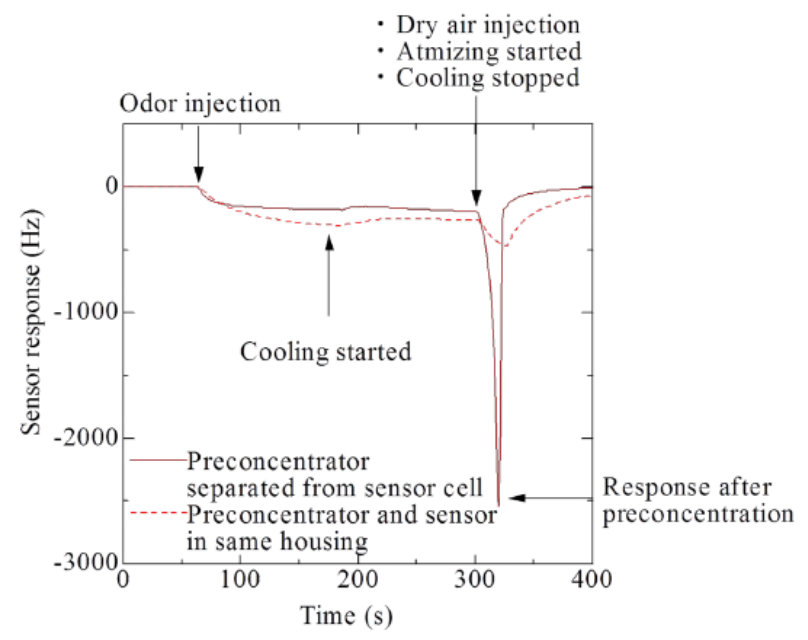

Fig.6 Response of QCM sensor to 1-butanol after preconcentration.

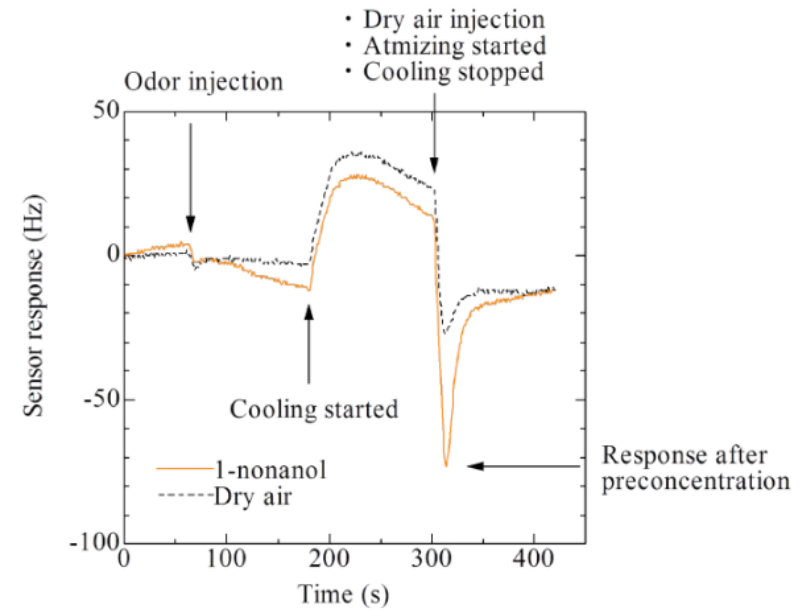

Fig.7 Response of QCM sensor to 1-nonanol after preconcentration.

For 1-butanol (1000 ppm), the behavior of this device was investigated. This result is shown in 
Figs. 6. The duty ratio of RF burst signal was $1 \%$. Initially, dry air was delivered to the preconcentrator cell. After $60 \mathrm{~s}, 1$-butanol was injected. After $180 \mathrm{~s}$, peltier device was turned on to cool the SAW device. The cooling time was 120s. Then, the SAW device was driven to atomize the condensed sample on the SAW substrate. Fig. 6 shows a data of QCM sensor's response after concentrating 1-butanol using preconcentrator separated from sensor cell and the cell with both preconcentrator and the sensor. This result indicates the performance of the latter is superior to the former.

Fig. 7 shows 1 -nonanol, one of low-volatile compounds, was concentrated by the cell with both preconcentrator and the sensor. This result indicates this device can concentrate 1 nonanol even with low concentration since the response to a preconcentrated odor around 300 $s$ was much higher than that without preconcentration around $60 \mathrm{~s}$.

\section{References}

[1] T.C.Pearce, S.S.Schiffman, H.T.Nagle and J.W.Gardner, Eds., Handbook of Machine olfaction, Weinheim, Germany: Wiley-VCH, 2003.

[2] K.Ema, M.Yokoyama, T.Nakamoto, and T.Moriizumi: Odor sensing system using quartzresonator sensor array and neural-network pattern recognition、Sensors and Actuators, 13(1989)291-296.

[3] Grate, J. W.; Rose-Pehrsson, S. L.; Venezky, D. L.; Klusty, M.Wohltjen, H. Smart sensor system for trace organophosphorus and organosulfur vapor detection employing a temperaturecontrolled array of surface acoustic wave sensors, Anal. Chem. 1993, 65, 1868

[4] Y.Sasaya and T.Nakamoto, Study of halitosissubstance sensing at low concentration using an electrochemical sensor array combined with a preconcentrator, IEE of Japan, 126 (2006) 292296.

[5] M. Kurosawa and T. Watanabe and A. Futami and T. Higuchi: "Surface acoustic wave atomizer", Sensors and Actuators A, Vol.50, No.1-2, p.69-74 (1995)

[6] A.Renaudin, P. Tabourier, V. Zhang, J.C. Camart, C. Druon: "SAW nanopump for handling droplets in view of biological applications.", Sensor and Actuators B, Vol.113, No.1, p.389-397(2006).

[7] "Handbook of Chemistry and physics", CRC Press 91st Edition.

[8] M. Devos, F. Patte, J. Rouault, P. Laffort : "Standardized Human Olfactory Thresholds", J. Odor Research and Eng., Vol.71, No.1, p.44-49(1991).
The frequency of QCM sensor changed due to the temperature change after 180s. However, it responded to 1-nonanol after 300 s since the frequency change under the dry air was small.

In Fig.7, the surface of SAW device was cooled down to $-5^{\circ} \mathrm{C}$ when a single peltier device was used. However, it was possible to cool it down to the temperature less than $-18^{\circ} \mathrm{C}$, which corresponds to human olfactory threshold in Table 1, when three peltier devices were used. Thus, the preconcentrator using SAW device is very promising.

\section{Conclusion}

The soundness of the basic idea of the preconcentrator using cooled SAW device was confirmed in this study. The further sensitivty enhancement is feasible when the temperature of the SAW device is further decreased. 